\title{
The Load Bearing CAPACity of RaIlWay MasonRy ARCH BRIDGES
}

\author{
Marek VOKAL, Michal DRAHORAD \\ Department of Concrete and Masonry Structures, Faculty of Civil Engineering, CTU in Prague, Thakurova 7, \\ 16629 Praha 6, Czech Republic \\ marek.vokal@fsv.cvut.cz,michal.drahorad@fsv.cvut.cz
}

DOI: $10.31490 /$ tces-2018-0019

\begin{abstract}
This paper deals with the way of calculating the load-bearing capacity of masonry arch railway bridges. It reviews the basic aspects of structural behaviour of these bridges, such as material non-linearity of masonry and interaction with the soil. Paper shows, how to include second order analysis in the calculation, because in some cases it might have non-negligible influence. It reminds the requirements of standards and shows, how to calculate the load-bearing capacity in accordance with these requirements with influence of mentioned non-linearities.
\end{abstract}

\section{Keywords}

Load-bearing capacity, masonry, material nonlinearity, vault.

\section{Introduction}

When evaluating existing bridges, the requirement for sufficient mechanical resistance and stability is expressed by the maximum load that the structure is able to transfer safely - the parameters of the rail vehicle which can go across the bridge under the defined conditions, ie the load bearing capacity. Determining the load bearing capacity of the existing bridge is governed by similar principles as the design of new structures (see applicable technical standards and regulations EN, DIN, TKP SŽDC and MVL). In the case of masonry vault structures this task is complicated by the structural behaviour and by the typical property of the material - a very small tensile strength. Due to these facts, the procedures for determining the load bearing capacity leads to non-linear analysis, which must consider a large number of frequently variable parameters. It is therefore very difficult to set up a simple analytical model and, for modelling of usual conventional structures, therefore, special programs developed directly for vault structures are often used.

The normal load bearing capacity of the bridge $Z_{L M 71}$ (hereinafter referred to as "load bearing capacity") is a dimensionless variable which expresses the ratio of the vertical effects of the variable load by rail transport (in terms of the relevant ultimate or serviceability limit state) to the effects caused by the load model 71 on the bridge object.

\section{Structural behavior}

\subsection{Behaviour of masonry structures}

For performing an analysis and design of the structure, the material - mortar and masonry elements - is homogenized in a suitable manner in such a way as to preserve its properties in relation to the real behaviour of the structure or its part. It is assumed that the dimensions of the masonry elements and joints between them do not significantly affect the distribution of stress in the brick element. The real stress-strain diagram of the masonry shows non-linear behaviour, particularly due to weak tensile strength - see [1]. In this work, it is considered that the material acts only in compression and when the tensile stress occurs, cracks occur and open up. If, subsequently, (e.g. in another load combination) the tension stresses in the cross section disappear, the cracks close and the cross-section acts again as full. The stress-strain diagram is considered linear in compression, the introduction of a non-linear diagram is not decisive from the engineering point of view at this stage of the research. 


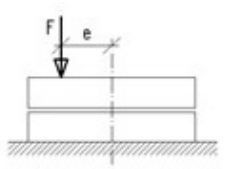

COMIRESSION

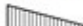

TENSION

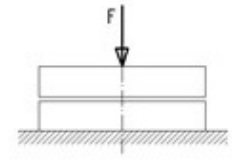

COMPRESSION

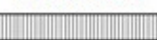

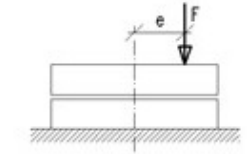

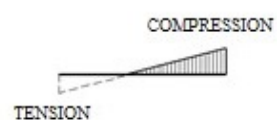

Fig. 1: Mechanism of change of cross-sectional characteristics and geometry of structure due to loading.

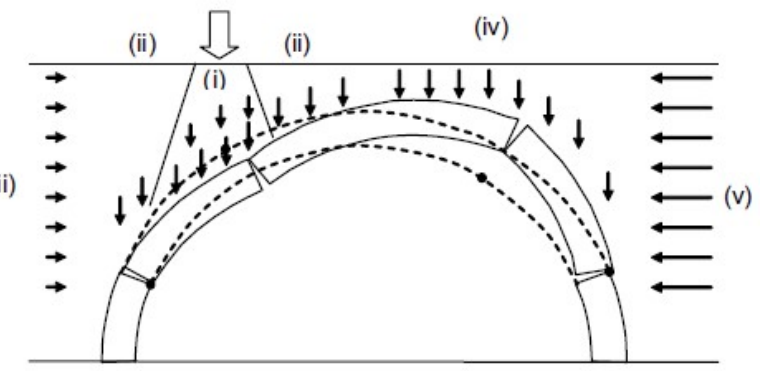

Fig. 2: Scheme of the actions of the backfill on the vault (i distribution of the loading load, ii and iv - the weight of the backfill material, iii - the active earth pressure at the deformation point of the vault away from the backfill, $\mathrm{v}$ - the passive pressure at the point of deformations of the vault into the backfill).

\subsection{Backfill of the vault and its interaction with the vault}

The backfill of the vault is an integral part of the structure. The effects of backfill on a vault can be divided into several basic kinds (see also Fig. 2 and [4] ):

- the distribution of concentrated loads from axles of load model into the back side of the vault, which reduces the local loads of the vault structure,

- self-weight,

- active ground pressure acting at points, where the vault deforms away from the backfill; the pressure decreases with the increasing separation of the vault from the backfill,

- passive earth pressure acting at the points of the construction, where the vault moves to the backfill and is therefore stabilizing structure; the pressure is increased by pushing the construction into the backfill.

The material used in the backfill is important, because it determines the behaviour of the entire structure. Usually, local materials were used to backfill of the arch, incoherent materials were preferred.

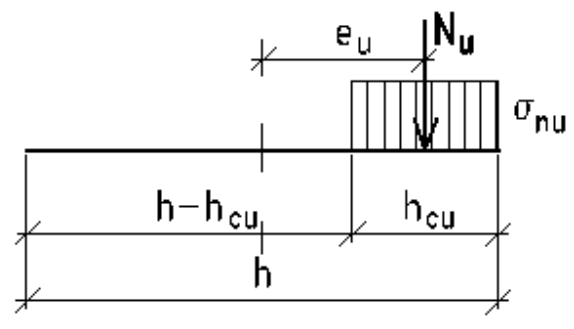

Fig. 3: Stress distribution of masonry cross-section at the ultimate limit state.

\subsection{Ultimate limit state}

The ultimate limit state (ULS) describes the behaviour of the structure in the state just before collapse, i.e. on the bearing capacity (load factor equals to 1.35 for dead load and 1.45 for live load). It is generally assumed that the structure is in a plastic state, there is a significant material damage and the full development of plastic joints. Conditions of resistance of the structure under a given applied load are then expressed according to 2] as:

$$
N_{R d}=f_{d} b\left(h-2 e_{u}\right)
$$

$$
V_{R d}=\left(f_{v k 0}+0.4 \sigma_{d}\right) b\left(h-2 e_{u}\right) / \gamma_{M} .
$$

$f_{d}$ is the design strength of masonry in compression, $f_{v k 0}$ is the characteristic value of initial shear strength at normal stress equal to $0, b, h$ is width and height, respectively, $e_{u}$ is the eccentricity of the resultant pressure force in cross-section in the ultimate limit state, $\sigma_{d}$ is the compressive stress in the compressed area at the ultimate limit state, $m$ is the coefficient of friction in the masonry joint, $\gamma_{M}$ is the factor of the material. The distribution of stress in the cross-section is considered constant, see Fig. 3 .

\subsection{Serviceability limit state}

The serviceability limit state (SLS) describes the behaviour of the structure under usual operating conditions. Fulfilling the conditions of serviceability limit state provides the required properties of the structure throughout its lifetime. In terms of serviceability limit state, crack width and structural stress under usual load are verified (load factor equals to 1.0). In terms of verification of vault structures, it is necessary to verify the maximal stresses in the cross-section and the height of the compressed area of the cross-section according to ČSN EN 1996-1-1. The behaviour of the cross-section is considered elastic with a linear distribution of stress in the compressed part of the cross section and excluded tensioned part (see Fig. 4). 


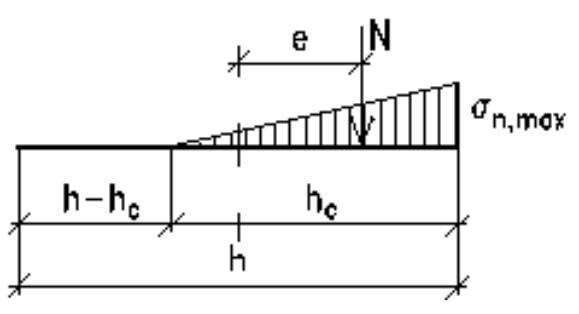

Fig. 4: Stress distribution of masonry cross-section at the serviceability limit state.

$$
\begin{gathered}
\sigma_{n, \max }=\frac{N_{E k}}{3 b(h-2 e)} \leq 0.45 f_{k} \\
h_{c} \geq \frac{h}{2} \longrightarrow e \leq \frac{h}{3} \\
e=\frac{M_{E k}}{N_{E k}}+e_{\text {init }}=\frac{M_{E k}}{N_{E k}}+\frac{L_{C r}}{450}
\end{gathered}
$$

$L_{C r}$ is critical buckling length, $M_{E k}$, is moment caused by load, $N_{E k}$ is normal force caused by load.

\section{Ways of modelling}

\subsection{General assumptions for calculation of program LimitState: RING and this study}

- The maximum span of the bridge $L$ is $20 \mathrm{~m}$.

- Minimal arch sagitta $f$ is $L / 6$ (not suitable for very flat arches and especially their middle parts).

- The structure must have a backfill.

- The thickness of the backfill is less than $L / 2$ (providing the dominant influence of the variable load).

- The masonry must have at least approximately perpendicular joints.

- The impact of parapet and spandrel walls is neglected.

\subsection{Content of this study}

The load bearing capacity of the bridges with spans ranging from 2.5 to $10 \mathrm{~m}$ has been calculated in the criterions of ULS and SLS. Three different relevant vault thicknesses, masonry strengths 2, 4, 6, 8 and $10 \mathrm{MPa}$, the sagitta $L / 2$ and $L / 4$ and height of the backfill 0.5 , 1.0 and $1.5 \mathrm{~m}$ were considered. These properties are
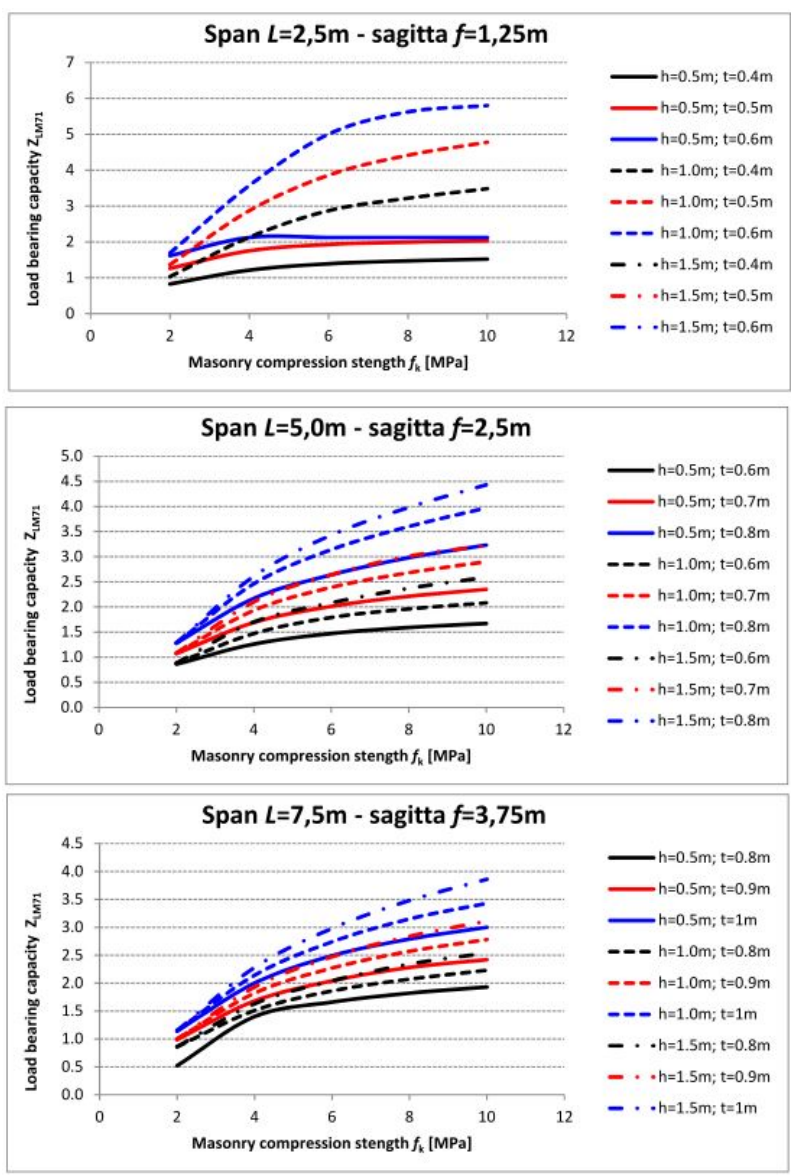

Fig. 5: Plot of load bearing capacity versus compressive strength of masonry in ULS.

common in these stuctures according to authors' experience (authors have already calculated tens of load bearing capacities of existing arch bridges). For the study of structures in ULS, the vaults were modelled in the LimitState: RING program and for the study of SLS the vaults were modelled in program Scia Engineer. In addition, a Matlab script was created, which calculates the deformations and internal forces on the vault structure including the effect of mentioned nonlinearities. The calculation is iterative, and at the occurrence of tensile stresses, the cross-sectional area is reduced, and thus the geometry of the structure changes in the next calculation step. The algorithm iterates until the calculation converges to the equilibrium position and the geometry of the structure does not change. See chapter 3.5. Since the modelling in the program LimitState: RING is less time-consuming than other methods, it was the task of this study to determine which of the limit states is decisive and to determine the value of the load bearing capacity according to the requirements of ULS, in which the load factor according to the requirements of $\operatorname{SLS}\left(Z_{1}\right)$ is equal at least one. 


\subsection{ULS}

LimitState: RING is a program that checks the load bearing capacity of the vault in the plane of the longitudinal section of the bridge structure, including the load distribution by the backfill. It uses equilibrium conditions on the parts of vault as a rigid bodies, the structure is divided into rigid bodies depending on creating the plastic hinges in locations with the lowest height of compressed area. It addresses also other influences which are not taken into account in this study: possible displacements of supports, true geometry, weakening of joints by the extraction of mortar and multi-span vaults. The disadvantage of the program is, that it does not consider the strength failure of the arch. The result of study using LimitState: RING can be seen in Fig. 5. For detailed results see [3].

\subsection{SLS - Scia Engineer}

To write a study on checking the serviceability limit state with different parameters of vaults, separate models for each vault was created in program Scia Engineer. The individual masonry elements were modelled by $2 \mathrm{D}$ elements - plane stress/plane strain, joints were modelled as beam members - 10 elements across the thickness of the vault. These beam members were entered a non-linearity "compression only". This has resulted in the masonry behaviour according to chapter 2.1. To consider the behaviour of the soil, the backfill was modelled also by the 2D element, with the behaviour considered linear. Since the program Scia Engineer cannot perform a non-linear calculation together with the calculation of the moving load, manual loading by force and continuous load of the model 71 in several (about 10) loading states with different load positions were always entered. Then the load factor was changed manually until either the limiting stress was reached in one of the bars representing the joint - Eq. 3. or there was a tension in the eleventh control beam which is in the middle of the height of each joint - this would mean breaking the requirement according to Eq. 4. This factor was recorded in the graph - see Fig. 8 and 9 .

\section{5. $\quad$ SLS - Matlab}

The script in Matlab model the arch with beam elements. The vault support was for this study chosen at both ends of the arch pinned. The backfill is modelled as a media, in which the load is distributed. Just as in the LimitState: RING program, the Bousinesq distribution is considered - see Fig. 11 .

According to the distribution angle in the soil, it is determined which elements are loaded by the axle of load model and the vertical load intensity at the par-
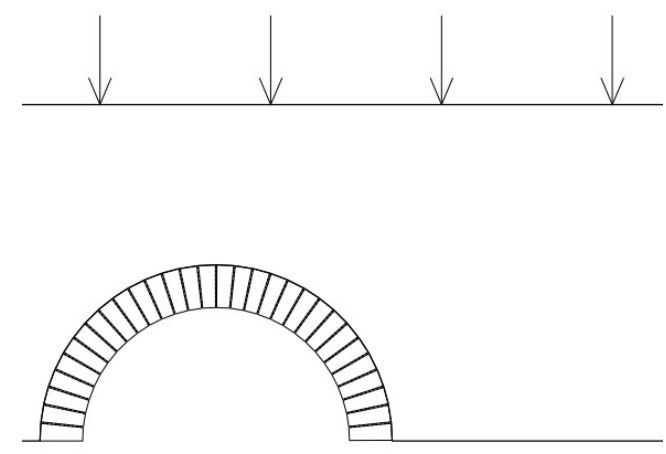

Fig. 6: Global model of the structure.

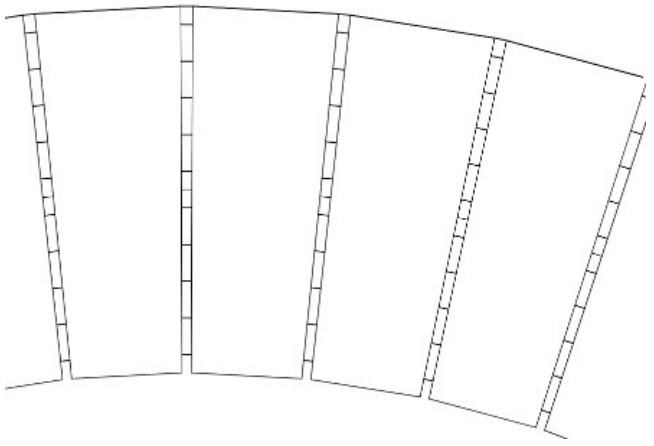

Fig. 7: Detail of the joint - ten beam elements across the height of the joint and eleventh in the middle.
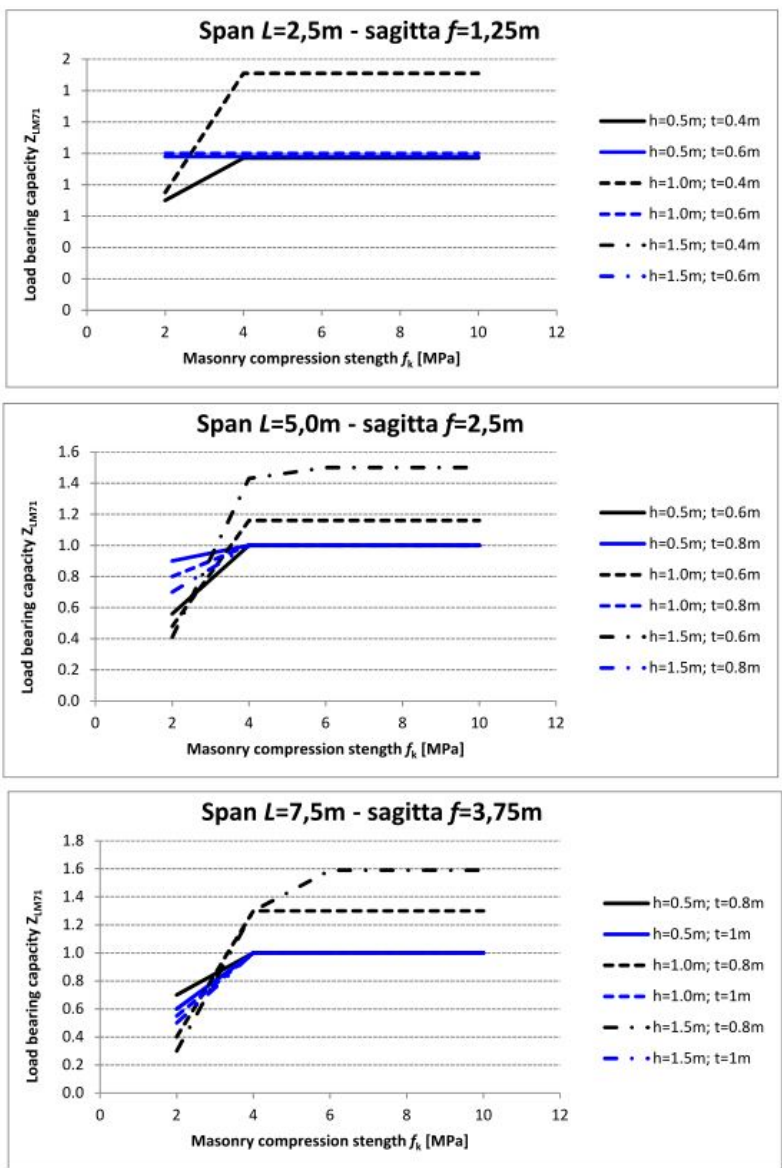

Fig. 8: Plot of load bearing capacity versus compressive strength of masonry in SLS for $f=L / 2$. 

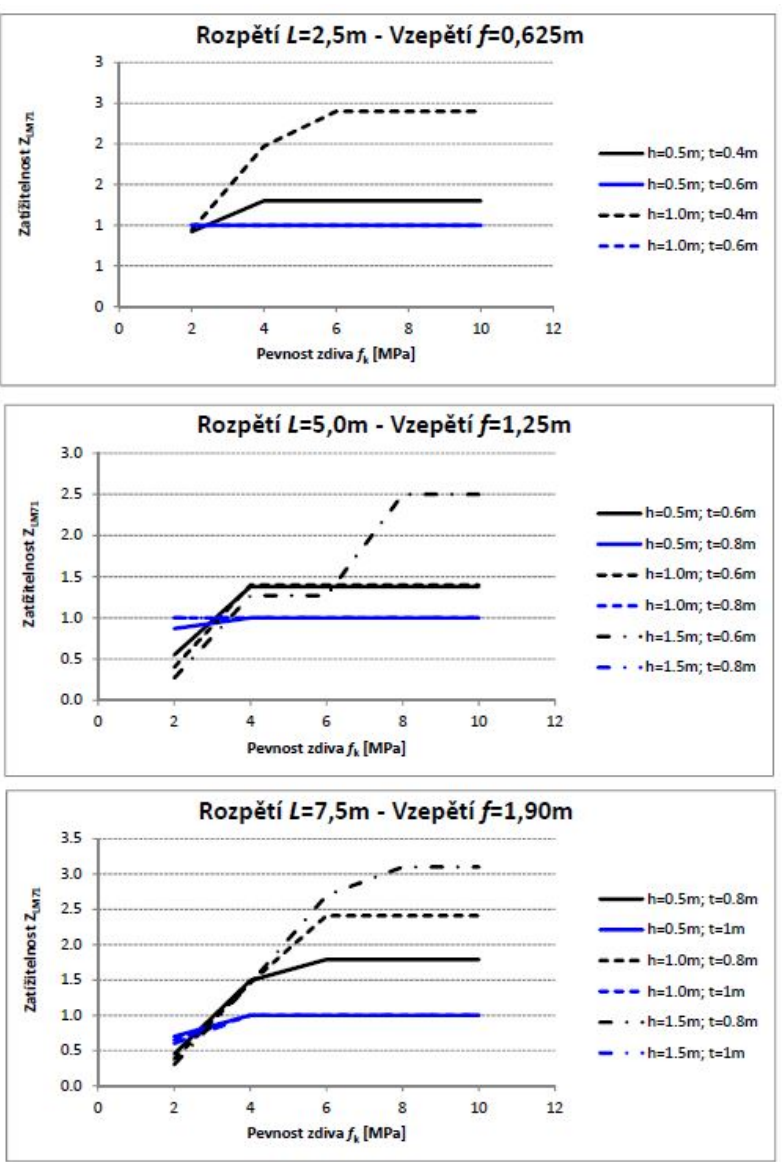

Fig. 9: Plot of load bearing capacity versus compressive strength of masonry in SLS for $f=L / 4$.

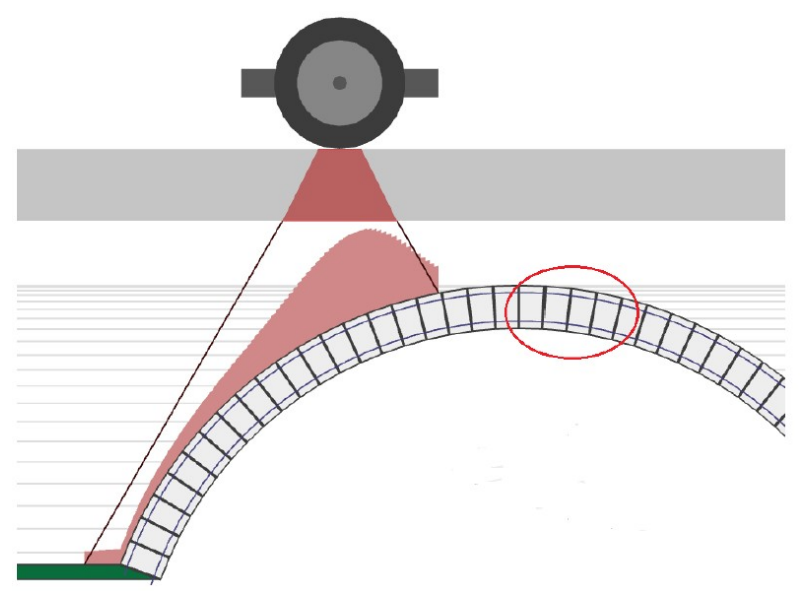

Fig. 10: Distribution of load.

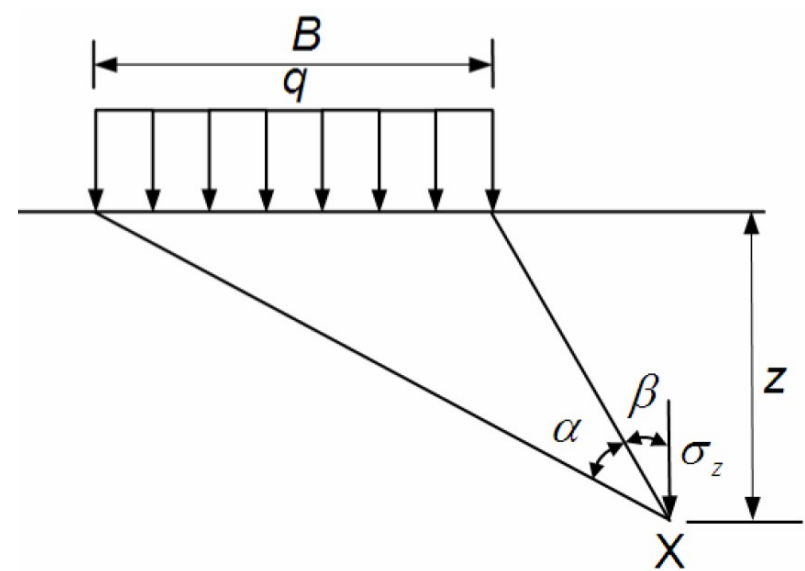

Fig. 11: Vertical load intensity in X location.

ticular node of the structure is determined according to the following relationship:

$$
\sigma_{z}=\frac{q}{\pi}[\alpha+\sin \alpha \cos (\alpha+2 \beta)] .
$$

$q$ is line continuous load on the surface and the angles $\alpha$ and $\beta$ see in the Fig. 11 .

The backfill is also modelled by horizontal springs to prevent the structure from the deforming to the backfill, thus transmit only the pressure. Their stiffness is chosen as the size of $E_{d e f}$ - modulus of deformation of the soil multiplied by the horizontal projection of the element. The magnitude of the force they can transfer is in reality limited by the passive pressure, for the purposes of this script it is not limited for simplicity, (in the serviceability limit state there must never be any increased deformations in which the passive pressure is activated). Given the nature of the problem (above mentioned) solving is performed by method of inverse iterations. This method is explained in [5]. The system of equations of the following form is solved:

$$
\left(\mathbf{K}-\mathbf{K}_{\mathbf{G}}\right) \mathbf{r}=\mathbf{0} .
$$

$\mathbf{K}$ is global stiffness matrix considering the boundary conditions (regular matrix), $\mathbf{K}_{\mathbf{G}}$ is a geometric stiffness matrix and $\mathbf{r}$ is a vector of displacement. Geometric stiffness matrix for a beam hinged on both sides is derived from a simple equilibrium conditions - see Fig. 12

$$
\mathbf{K}_{\mathbf{G}}=\frac{N}{l}\left[\begin{array}{cccccc}
0 & 0 & 0 & 0 & 0 & 0 \\
0 & 1 & 0 & 0 & -1 & 0 \\
0 & 0 & 0 & 0 & 0 & 0 \\
0 & 0 & 0 & 0 & 0 & 0 \\
0 & -1 & 0 & 0 & 1 & 0 \\
0 & 0 & 0 & 0 & 0 & 0
\end{array}\right]
$$

$N$ is normal force in the beam and $l$ is a beam length. 


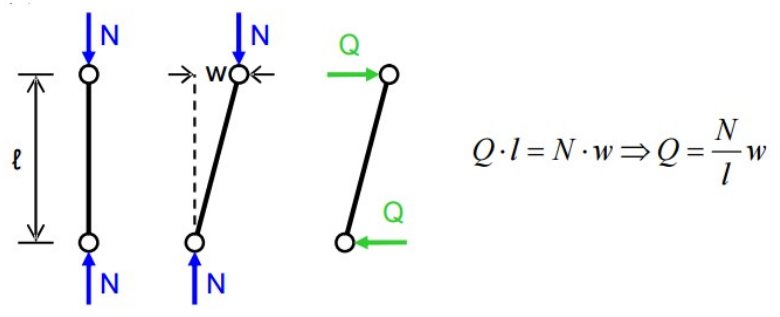

Fig. 12: Derivation of geometric stiffness matrix for a beam hinged on both sides.

Geometric stiffness matrix for a beam fixed on both sides:

$$
\mathbf{K}_{\mathbf{G}}=\frac{N}{l}\left[\begin{array}{cccccc}
0 & 0 & 0 & 0 & 0 & 0 \\
0 & 36 & 3 l & 0 & -36 & 3 l \\
0 & 3 l & 4 l^{2} & 0 & -3 l & -l^{2} \\
0 & 0 & 0 & 0 & 0 & 0 \\
0 & -36 & -3 l & 0 & 36 & -3 l \\
0 & 3 l & l^{2} & 0 & -3 l & 4 l^{2}
\end{array}\right] .
$$

Equation 7 can be modified for the purpose of calculation to:

$$
\mathbf{K r}=\mathbf{f}+\mathbf{f}_{\text {ekv }} .
$$

$\mathbf{f}$ is a load vector, and $\mathbf{f}_{\mathbf{e k v}}$ is vector of equivalent load calculated as follows:

$$
\mathrm{f}_{\mathrm{ekv}}=\mathrm{K}_{\mathrm{G}} \mathbf{r} \text {. }
$$

In the $(i+1)-$ th step of iteration we can calculate:

$$
\mathbf{K} \mathbf{r}^{\mathbf{i}+\mathbf{1}}=\mathbf{f}+\mathbf{K}_{\mathrm{G}}^{\mathbf{i}} \mathbf{r}^{\mathbf{i}}
$$

$i$ is number of step. It is used in upper indices. Analysis uses following algorithm:

$$
\begin{gathered}
\mathbf{K} \mathbf{r}^{\mathbf{0}}=\mathbf{f} \\
\mathbf{K} \mathbf{r}^{\mathbf{i}+\mathbf{1}}=\mathbf{f}+\mathbf{K}_{\mathbf{G}}^{\mathbf{i}} \mathbf{r}^{\mathbf{i}} \\
\left.\| \mathbf{r}^{\mathbf{i}+\mathbf{1}}-\mathbf{r}^{\mathbf{i}}\right) \|<\epsilon .
\end{gathered}
$$

Equation 13 is solved first to obtain $r^{0}$, from which $N^{0}, \mathbf{K}_{\mathbf{G}}^{\mathbf{0}}$ are calculated. Then, from Eq. 14 we obtain $r^{i+1}$ - vector of displacement of next step. This step is repeated until the criterion of Eq. 15 is fulfilled. In Matlab algorithm, error is chosen to be smaller than $\epsilon=10^{-8} \mathrm{~m}$. As an example, the vault with the thickness $0.5 \mathrm{~m}$, diameter $5 \mathrm{~m}$ and sagitta $1.9 \mathrm{~m}$ was calculated. It was loaded by self-weight and axles of load model LM71. The height of backfill at the top of vault is considered $2 \mathrm{~m}$. The result is shown by Fig. 13,14 and 15.

The calculation converged within eight steps.

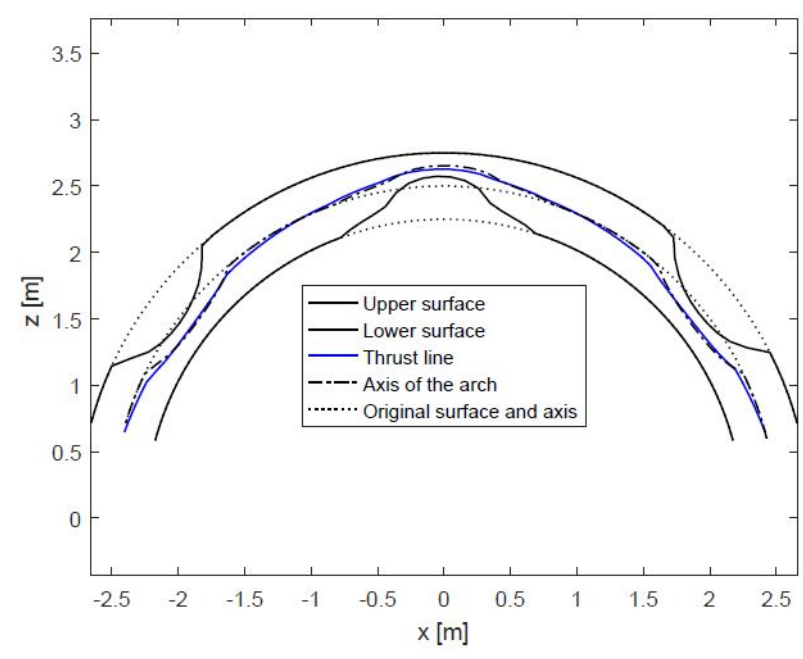

Fig. 13: The resulting thickness of the compressed part of structure when loaded by the self-weight of the structure and the backfill.

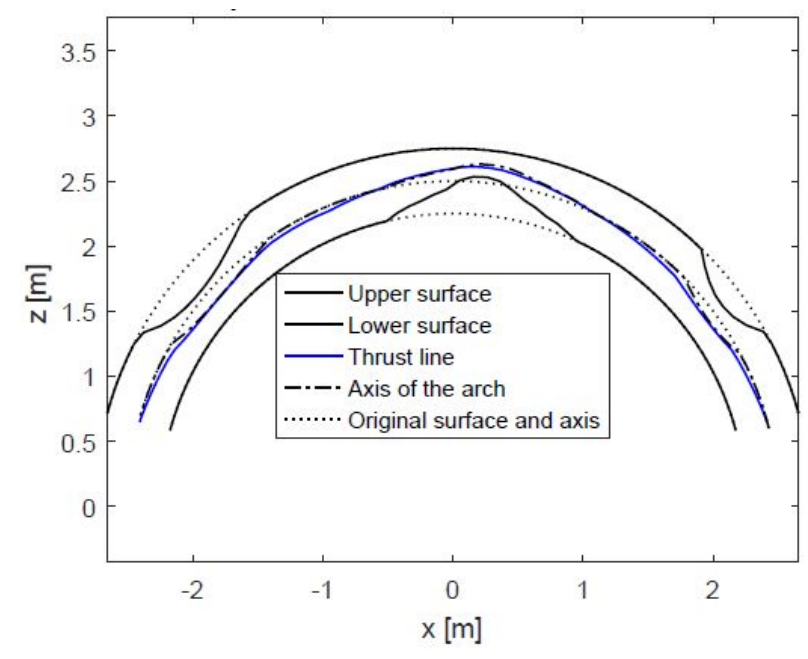

Fig. 14: The resulting thickness of the compressed part of structure under the load of the axles of the LM71 in the left half of the vault.

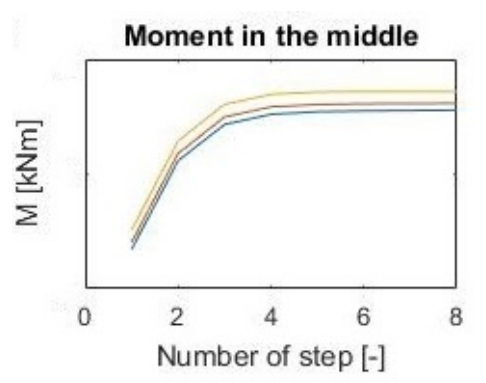

Fig. 15: The resulting bending moment on the three elements approximately in the middle of the arch in the individual calculation steps. 


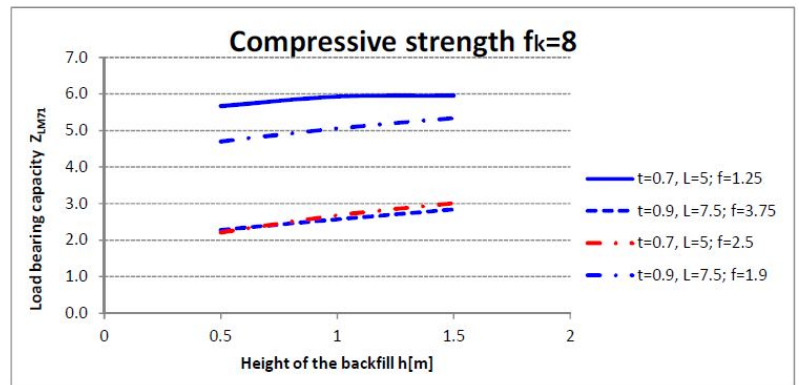

Fig. 16: Plot of load bearing capacity versus height of backfill in SLS.

\section{Discussion of the result}

\subsection{Scia Engineer}

For the various selected values of sagitta $f$, height of the backfill $h$ and strength of masonry $f_{k}$, a plot of load bearing capacity versus height of backfill was constructed - see Fig. 16

Generally, it can be read out from the graphs (but only in the range of parameters mentioned in chapter 3.1.), that:

- at lower sagitta, the vault has a higher load bearing capacity,

- for a higher backfill, the vault has a higher load bearing capacity,

- for a higher vault thickness, the vault has a higher load bearing capacity.

The total number of $Z_{1}$ coefficients that are larger than 1 in this study is 32 . The value varies from 1 to 3.22 . From Fig. 8 and 9 it can be seen that the most tough of all the criteria of limit states considered is the criterion according to Eq. 3 for the low-strength of the masonry (2-4 MPa), which determines the maximum admissible pressures in the masonry, while for the vaults with a higher strength is the load bearing capacity limited by the criterion according to the Eq. 4 determining the maximum eccentricity of the resultant force in the cross section.

\subsection{Matlab}

One vault with one axle position was verified in the Matlab script. To obtain the results of a study of multiple vaults of different parameters, another Matlab script must be created, that will call the original script many times with different parameters and store the results different load positions and different parameters of vaults. So far, we can say that the results obtained so far are real and that it is easy to distinguish whether the loaded structure fulfils the requirements after finishing the calculation. Only the thickness, diameter and arch of the vault, the characteristic strength of the masonry and the height of the backfill at the top of vault are specified by user in the script. Entering this data is therefore very operative and fast. Until now, only circular arches hinged on both sides were verified, the author can modify the shape of the vault, for example on an ellipse or other shape, or to modify the support to fixed or elastic.

\section{Conclusion}

A study has been carried out showing how masonry vault structures behave and how they should be analysed. It compares which of the limit states - ULS or SLS - is more tough and therefore results in lower load bearing capacity of masonry vault railway bridges. It has revealed that the requirements set by SLS are tougher than ULS requirements. It also showed the general rules, how the differing parameters (thickness, sagitta, height of the backfill) influence the load bearing capacity.

\section{References}

[1] DRAHORAD, M. Load-Bearing Capacity of Masonry Arch Bridges. International Conference Engineering Mechanics . 2013, vol. 19, iss. 1, pp. 3334. ISSN 1802-1484.

[2] EN 1996-1-1. Design of masonry structures. ÚNMZ, 2005.

[3] DRAHORAD, M. The guide to use of software LimitState: Ring for employees of SŽDC.

[4] LimitState:RING Manual. Available at: http://www.limitstate.com/files/ pdf/ring/RING_User_Manual.pdf

[5] Linear stability and theory of II. Order. Available at: http://mech.fsv.cvut.cz/ leps/ teaching/ankc/cviceni_08.pdf 